\author{
Roundness Measurement of Spherical Artifacts at Arbitrary Latitude \\ Eric Marsh ${ }^{1 *}$, Jeremiah Couey ${ }^{1}$, Ryan Vallance ${ }^{2}$ \\ ${ }_{1}^{1}$ The Pennsylvania State University, 21 Reber Building, University Park, PA 16802, USA \\ ${ }^{2}$ The George Washington University, 738 Phillips Hall, 801 22nd Street N.W., Washington, DC 20052, USA
}

\begin{abstract}
This technical note examines an approach to measuring the roundness of spherical artifacts at arbitrary latitude. A roundness measurement includes the combined contributions from the artifact's form error and the spindle's error motion. We show that it is possible to separate the spindle error from artifact form error at any inclination with respect to the axis of rotation if the axial and radial error motion is independently measured using an error separation technique such as Donaldson reversal. Comparison of the predicted and measured results shows agreement to less than a nanometer.
\end{abstract}

Keywords: Spindle metrology, roundness metrology, reversal

\title{
Introduction
}

Figure 1 shows a spherical artifact with capacitive sensor locations for axial $(Z)$ and radial $(R)$ measurements and a general measurement $M$ taken at an arbitrary latitude $\phi$. At current limits of precision, the typical form error of a lapped spherical artifact is of the same order of magnitude as the error motion of the instrumentgrade spindles used in measurements. Evans, Hocken, and Estler reviewed the procedures that separate the contribution of the spindle's error motion from the form error of the artifact [1]. One method, Donaldson reversal, separates synchronous radial error motion from roundness profiles of the artifact when the sensor is located at the equator $\left(\phi=90^{\circ}\right)$. As will be shown, Donaldson reversal is not suitable for determining roundness at arbitrary latitude because both the axial and radial error motion contribute to the measurement yet they cannot be simultaneously reversed by moving the displacement indicator. As demonstrated in this note, measuring roundness at arbitrary latitudes requires independent knowledge of the spindle's axial and radial error motion at the equator. Once determined, the method presented in this note separates spindle error motion from artifact roundness profiles at any arbitrary location without additional reversals. The noncontact sensor at $M$ is separated from the artifact by the offset distance $g$, and the sensor measures changes in the standoff distance $\Delta$. The measured change $\Delta$ is due to fluctuations produced by spindle error motion and the artifact form error $P$.

Equations relating displacements measured by the sensor to the radial, axial, and tilt error motion are determined using the geometric model shown in Figure 2. We assume that the capacitive sensor, inclined at an angle $\phi$ with respect to the axis of rotation $\left(\phi=0\right.$ for an axial measurement and $\phi=90^{\circ}$ for a radial

*Corresponding author. Tel.: +814-865-5242; fax: +814-865-2986. E-mail address: emarsh@psu.edu. 
measurement), is only sensitive to displacement normal to the electrode surface. We also neglect any effect on the displacement measurement due to the form error since it is small compared to the effective spot size of the sensor.

For convenience, the origin of a Cartesian coordinate frame is located at the point in which the radial error motion of the spindle is zero (at some arbitrary location $d$ from the center of the spherical artifact). In the special case of a spindle with radial error motion but zero tilt error motion $(d=\infty)$, the analysis is slightly different but leads to an identical result.

A spherical artifact of radius $r$ is shown in two positions. The first position of the sphere is ideally centered at point $P_{1}$ on the axis of rotation. The second position includes axial error motion $Z$ and tilt error motion $\alpha$. Radial error motion is always associated with a specific location along the axis of rotation because of the tilt component [2]. The point $P_{3}$ is the point on the sphere that is closest to the sensor electrode when there is no spindle error motion (i.e., the sphere is centered at $P_{1}$ ). The coordinates of points $P_{1}, P_{2}$, and $P_{3}$ are then given by Eqs (1)-(3).

$$
\begin{gathered}
P_{1}=(0, d) \\
P_{2}=(d \sin \alpha, Z+d \cos \alpha) \\
P_{3}=(r \sin \phi, d+r \cos \phi)
\end{gathered}
$$

Next we consider four construction lines. The lines are defined such that $l_{1}$ passes through $P_{1}$ and is normal to the sensor. By definition, $P_{3}$ will be on this line. Line $l_{2}$ passes through $P_{2}$ and is also normal to the sensor. Line $l_{3}$ is parallel to the sensor surface and passes through point $P_{3}$. Finally, line $l_{4}$ is coincident with the end of the sensor. The slope of lines $l_{1}$ and $l_{2}$ is $\cot \phi$ while the perpendicular lines $l_{3}$ and $l_{4}$ have slopes of $-\tan \phi$. The equation for each line is given in Eqs (4)-(7).

$$
\begin{array}{ll}
\text { line } 1 & y_{1}=x \cot \phi+d \\
\text { line } 2 & y_{2}=x \cot \phi+d(\cos \alpha-\sin \alpha \cot \phi)+Z \\
\text { line } 3 & y_{3}=-x \tan \phi+r \sec \phi+d \\
\text { line } 4 & y_{4}=-x \tan \phi+(r+g) \sec \phi+d
\end{array}
$$

The capacitive sensor standoff distance $g$ is the distance from the sensor electrode to point $P_{3}$. For the second position of the sphere, the distance between the sensor and target is found using the intersection of lines $l_{2}$ and $l_{4}$. This point of intersection, $P_{4}$, is the point on the sensor that is closest to the sphere when the spindle error motion is included. The coordinates of $P_{4}$ are given in $\mathrm{Eq}(8)$.

$$
P_{4}=\left(\begin{array}{c}
d \sin \phi \cos \phi(1+\cot \phi \sin \alpha-\cos \alpha)+(g+r) \sin \phi-Z \sin \phi \cos \phi, \ldots \\
d\left(\cos ^{2} \phi-\sin \alpha \sin \phi \cos \phi+\cos \alpha \sin ^{2} \phi\right)+(g+r) \cos \phi+Z \sin ^{2} \phi
\end{array}\right)
$$


The distance between point $P_{2}$ and $P_{4}$ is subtracted from the nominal distance between the center of the sphere and the sensor $(r+g)$ to obtain the detected displacement $\Delta$ as shown in Eq (9). The detected displacement varies as a function of the spindle rotation angle $\theta$.

$$
\begin{aligned}
\Delta & =(r+g)-\overline{P_{2} P_{4}} \\
& =d(\cos (\alpha-\phi)-\cos \phi)+Z \cos \phi
\end{aligned}
$$

At this point, Eq. (9) is simplified by noting that the tilt error motion of the spindle $\alpha$ is a small angle in any precision spindle. We also note that by evaluating Eq. (9) at $\phi=0$ and $90^{\circ}$ we can relate the axial and radial error motion as determined by spindle measurements on axis and on the equator to the components of Eq. (9).

$$
\begin{gathered}
\Delta(\phi=0)=Z \\
\Delta\left(\phi=90^{\circ}\right)=d \sin \alpha=R
\end{gathered}
$$

Therefore, by the application of a trigonometric identity, the small angle approximation for $\alpha$, and the substitution of Eqs. (10) and (11) into Eq. (9) we find that the measured spindle error motion at any latitude $\phi$ is a simple combination of the axial and radial error motion.

$$
\Delta=R \sin \phi+Z \cos \phi
$$

In general, a measurement $M(\phi)$ made at latitude $\phi$ will include not only the spindle error motion $\Delta$ as considered above, but also the form error of the sphere $P(\phi)$.

$$
M(\phi)=R \sin \phi+Z \cos \phi+P(\phi)
$$

It is worth noting that Eq. (13) shows that Donaldson reversal cannot be directly applied to measurements made in the general case of latitudes other than 0 and $90^{\circ}$ because we now have three unknowns and two equations. Specifically, the unknowns at some latitude $\phi$ are the contribution of the axial error motion $Z$, the radial error motion $R$, and the artifact form error $P$. A Donaldson reversal performed at arbitrary latitude $\phi$ provides two equations, one for each measurement. Therefore, we cannot separate the artifact form error from the spindle at arbitrary latitude $\phi$ without at least first determining the axial error motion $Z$. In practice it may be more convenient to determine both $Z$ and $R$ by Donaldson reversal and then apply Eq. (13) just once.

\section{Experimental Results}

Experimental verification of the analysis was carried out on test hardware consisting of an unmotorized air bearing spindle (Professional Instruments, BLOCK-HEAD ${ }^{\circledR} 4 \mathrm{R}$ ) mounted on a precision rotary table (Moore Tool Company, LRT). The sensor was a capacitance probe (Lion Precision, C-1C, $0.5 \mathrm{~nm} / \mathrm{mV}$ ) and the 
spherical artifact was a Ø64 mm lapped steel reference sphere (Professional Instruments). The experimental procedure followed the B89.3.4M ANSI Standard and is further described by Grejda [3]. All data were lowpass filtered to 50 undulations per revolution.

Figure 3 shows data taken in the $\mathrm{X}$ and $\mathrm{Y}$ directions at $\phi=0,45^{\circ}$, and $90^{\circ}$. Data can be taken in both the $\mathrm{X}$ and $\mathrm{Y}$ directions to compute two independent estimates of the artifact form error for the sake of comparison. The data shown for $\phi=45^{\circ}$ and $\phi=90^{\circ}$ include both the spindle error motion and the artifact form error. As outlined in the preceding section, the spindle error motion $R(\phi=0)$ and $Z\left(\phi=90^{\circ}\right)$ is needed in order to determine the artifact's roundness at $\phi=45^{\circ}$. Therefore, two additional measurements (not shown) were made at the equator $\left(\phi=90^{\circ}\right)$ to perform Donaldson reversals in the $\mathrm{X}$ and $\mathrm{Y}$ direction. After determining $\mathrm{R}$ with these two reversals and $Z$ from a single axial measurement, $M(\phi)$ was calculated twice with Eq. (13). Ideally, the form error will be the same regardless of whether the measurements are made in the $\mathrm{X}$ or $\mathrm{Y}$ direction, which does turn out to be the case. Figure 4 shows the calculated form error at $\phi=45^{\circ}$ based on four separate tests: two in the $\mathrm{X}$ direction and two in the $\mathrm{Y}$ direction. The four estimates of the form error agree to better than 0.5 nanometers. The only post processing applied to these results is the 50 undulations per revolution low-pass filtering.

As a final test of this technique, it is possible to isolate the radial component of the spindle error motion using two measurements at latitude $\phi$ made $180^{\circ}$ apart in $\theta$, as in a Donaldson reversal. Measurement $M_{1}$ is made first and followed by $M_{2}$, which is made after the artifact and probe are indexed $180^{\circ} . M_{1}$ and $M_{2}$ include the combined effects of the spindle error motion and the artifact form error as shown in Eqs. (14) and (15).

$$
\begin{gathered}
M_{1}=R \sin \phi+Z \cos \phi+P \\
M_{2}=-R \sin \phi+Z \cos \phi+P
\end{gathered}
$$

The radial error motion is isolated by taking the difference between these two equations.

$$
\mathrm{R}=\frac{M_{1}-M_{2}}{2 \sin \phi}
$$

This computed radial error motion using Eq. 16 may be compared to the radial error motion measurement determined directly by Donaldson reversal, as shown in Figure 5. In other words, we are comparing the radial error motion of the spindle as determined by Donaldson reversal to the radial error motion of the spindle as computed from different measurements taken at a different latitude. These two approaches agree within 0.6 $\mathrm{nm}$, with the possibility of further improvement with future reductions in the uncertainty of the probe angle $\phi$. 


\section{Conclusion and Discussion}

This note introduces an approach to measuring artifact form error for spheres at arbitrary latitudes. The analysis of a simple geometric model shows that an error separation technique cannot be applied at latitudes other than 0 and $90^{\circ}$ without additional measurements. The method requires knowledge of the axial and radial spindle error motion as obtained by an error separation technique such as Donaldson reversal. The results show sub-nanometer repeatability and while no independent metrology technique is available to verify the results, the method is partially self-checking in that the radial error motion of the spindle at the equator can be predicted from measurements made at other latitudes. 


\section{Figures}

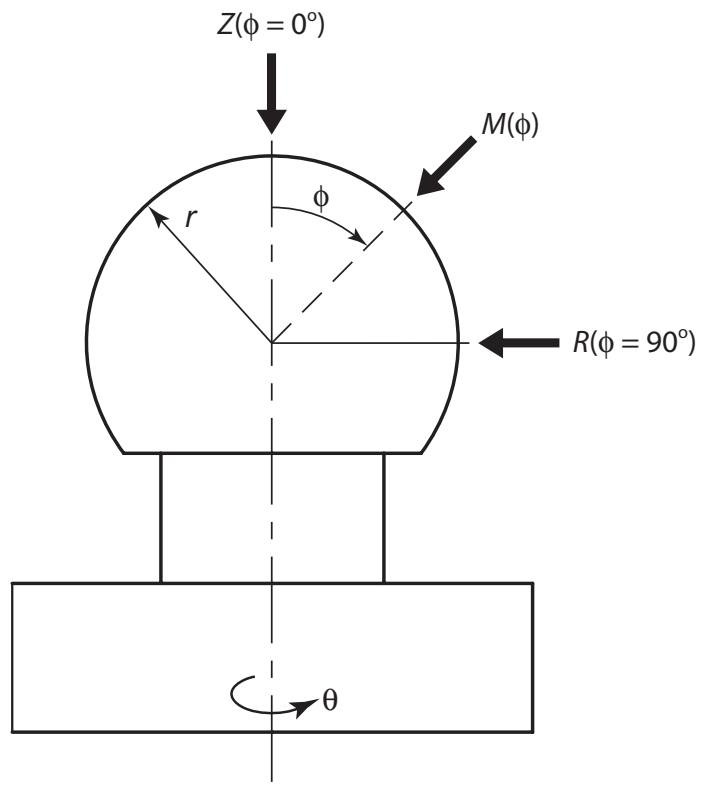

Figure 1 Schematic of a spherical artifact with axial, radial and arbitrary measurement locations.

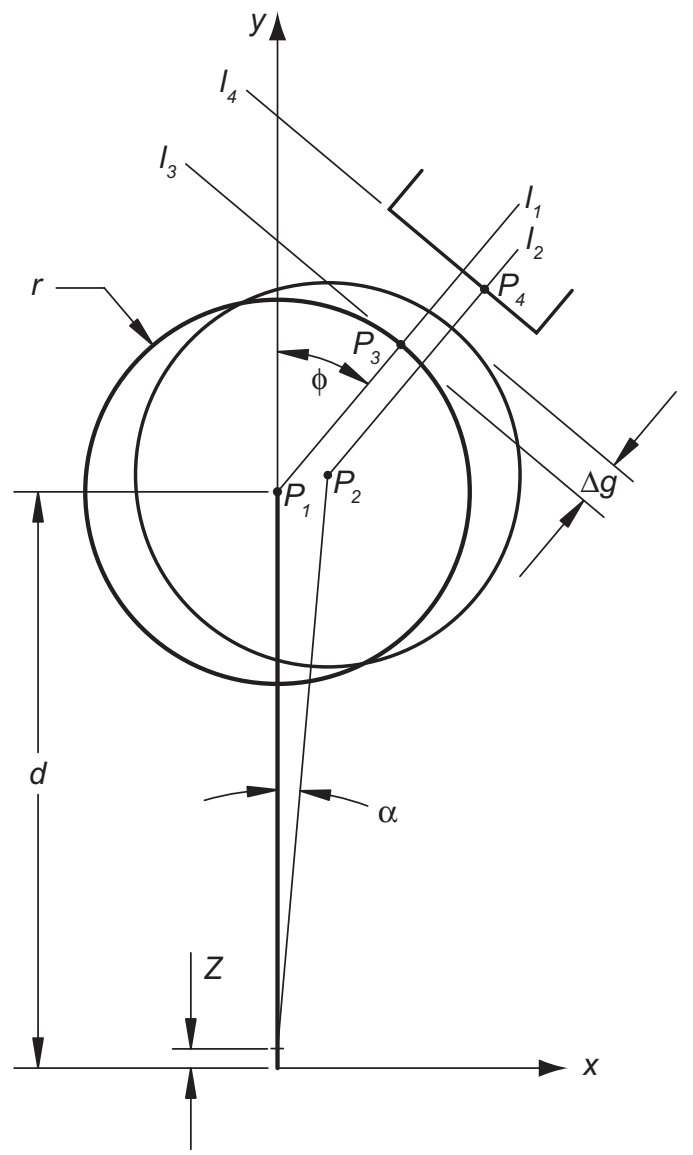

Figure 2 Geometric model used to determine the change in distance to the displacement sensor. 


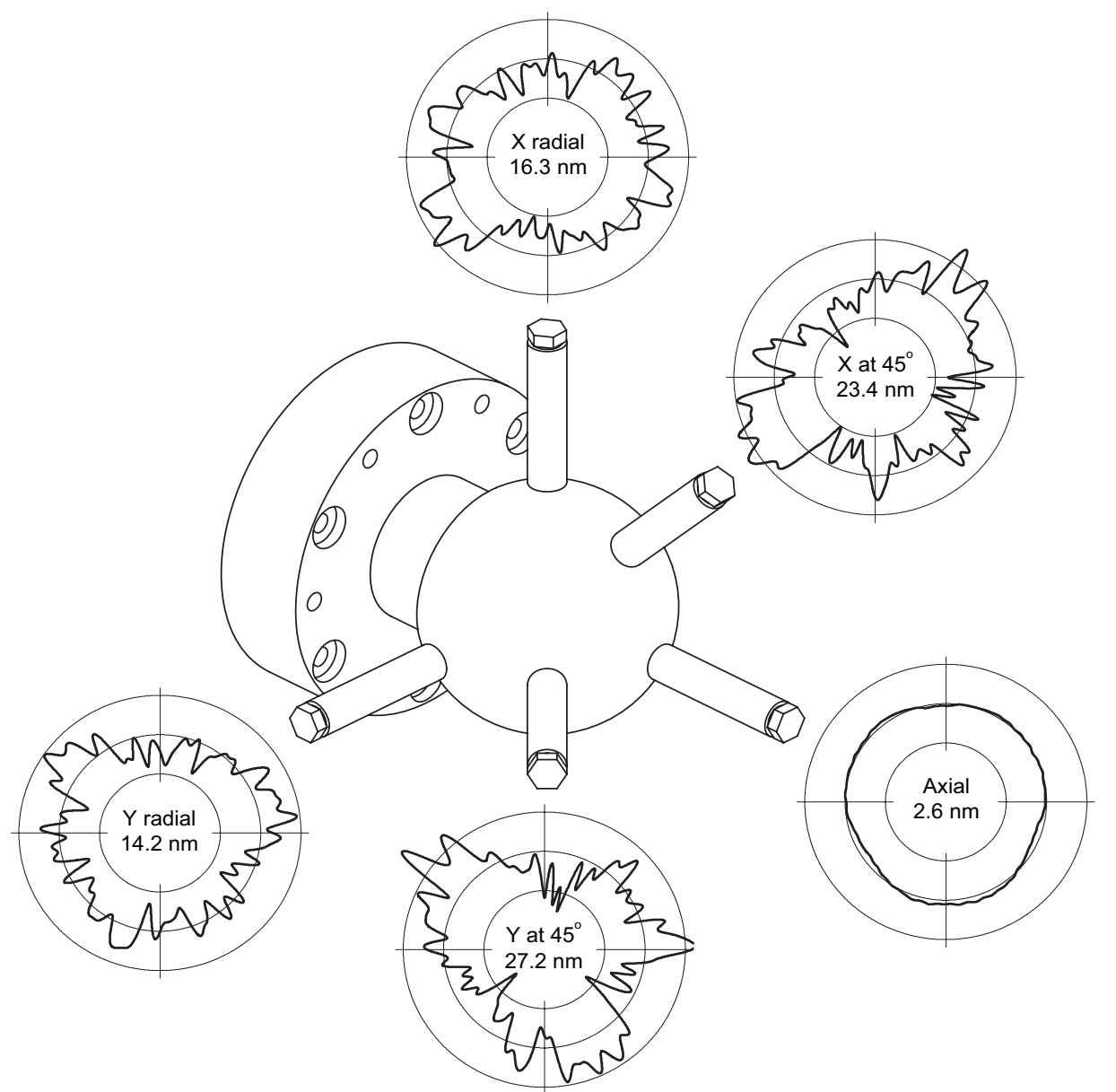

Figure 3 Measurements reflecting the combined contribution of spindle and artifact error. 


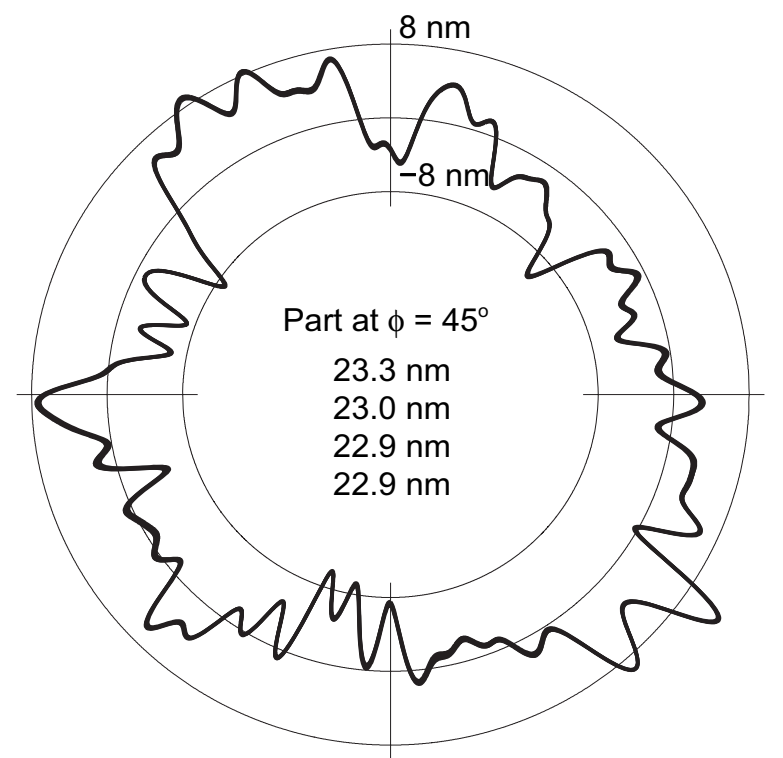

Figure 4 Four separate estimates of the artifact form error at $\phi=45^{\circ}$.

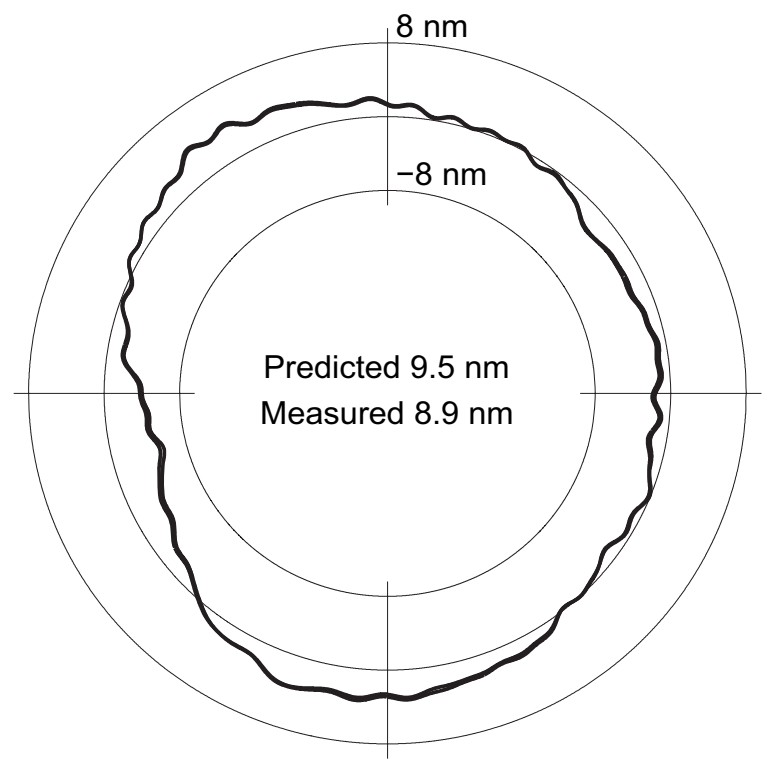

Figure 5 Comparison of the computed and measured radial error motion at the equator of the spherical artifact. 


\section{References}

1. Evans CJ, Hocken RJ, Estler WT. Self-Calibration: Reversal, Redundancy, Error Separation, and “Absolute Testing". CIRP, 45 (2), 1996; 617-634.

2. ANSI/ASME B89.3.4M Axes of Rotation: Methods for Specifying and Testing Standard, 1985.

3. Grejda RD. Ultra-precision Spindle Metrology, PhD Dissertation, Penn State University, 2002. 